\title{
ORTNER'S SYNDROME
}

\author{
Fábio Muradás Girardi ${ }^{1}$, Rodrigo Machado Ardenghi ${ }^{2}$
}

\begin{abstract}
Inferior laryngeal nerve palsy is a relatively common entity. Nevertheless, an occurrence as a result of a cardiovascular pathology is rare. In this case, it is called Ortner's syndrome (OS). Aortic diseases are responsible for more than half of cases. Supraaortic vessels disorders are rare causes of OS. In our new report, a non-smoker and non-drinker 70-year-old woman presented with a history of dysphonia since childhood. On direct laryngoscopy, a left vocal cord paralysis was detected and a contrast-enhanced computed tomography showed an aberrant right subclavian artery originating from the left portion of the aortic arch. Its course to its usual site runs behind the esophagus, being also called arteria lusoria. In this particular case, two unusual situations appear together, which contributes to the rarity of the event.
\end{abstract}

Keywords: Recurrent laryngeal nerve; vocal cord paralysis; hoarseness

Inferior laryngeal nerve palsy is a relatively common entity, although its true incidence remains under discussion. Around $1 / 4$ of tertiary care patients with a major complaint of dysphonia are diagnosed with vocal cord paralysis or paresis ${ }^{1}$. In most cases the palsy is a result of inadvertent injury during a surgical procedure or a consequence of invasion or compression by a malignant tumor. An occurrence as a result of a cardiovascular pathology is rare. In this case, it is called Ortner's syndrome (OS) or cardiovocal syndrome ${ }^{2}$. Many different abnormalities of the heart and vessels may be causing this syndrome. As the left nerve is longer, it is 1.75 times more affected than the right side.

In 2014 Yuan published a comprehensive review of all OS cases reported from 1980 to $2011^{3}$. In total, 245 cases were found, with a male-to-female ratio of $1.84: 1$ and a mean age of 53.9 years. Aortic diseases were responsible for more than half of cases, with aortic aneurysm being the most frequent problem found. Supraaortic vessels disorders were responsible for only $6.25 \%$ of cases. An aberrant right subclavian artery (also called arteria lusoria) was reported in only one case, with an associated aneurysm.

In our new report, a 70-year-old woman, non-smoker and non-alcohol drinker presented with a history of dysphonia since childhood, associated with occasional choking. She had no history of surgical procedures, significant past diseases, associated symptoms or signs. On direct laryngoscopy, a left vocal cord paralysis was detected. A contrast-enhanced computed tomography (CT) showed an aberrant right subclavian artery (Figure 1), originating distally to the origin of the left subclavian artery on axial view (Panel A) and on a coronal view (Panel B), with no signs of aneurysmatic dilatation. Its course to its usual site runs behind the esophagus on axial view (arrow, Panel C) and on sagittal view (arrow, Panel D). No abnormalities were found on neck CT. Despite the aberrant course, no dysphagia was present.
Clin Biomed Res. 2020;40(1):61-62

1 Centro Integrado de Oncologia, Hospital Ana Nery. Santa Cruz do Sul, RS, Brasil.

2 Departamento de Radiologia, Hospital Santa Cruz. Santa Cruz do Sul, RS, Brasil.

Corresponding author:

Fábio Muradás Girardi fabiomgirardi@gmail.com Thomaz Flores Street, 1173, 602. 96810-078, Santa Cruz do Sul, RS, Brasil. 


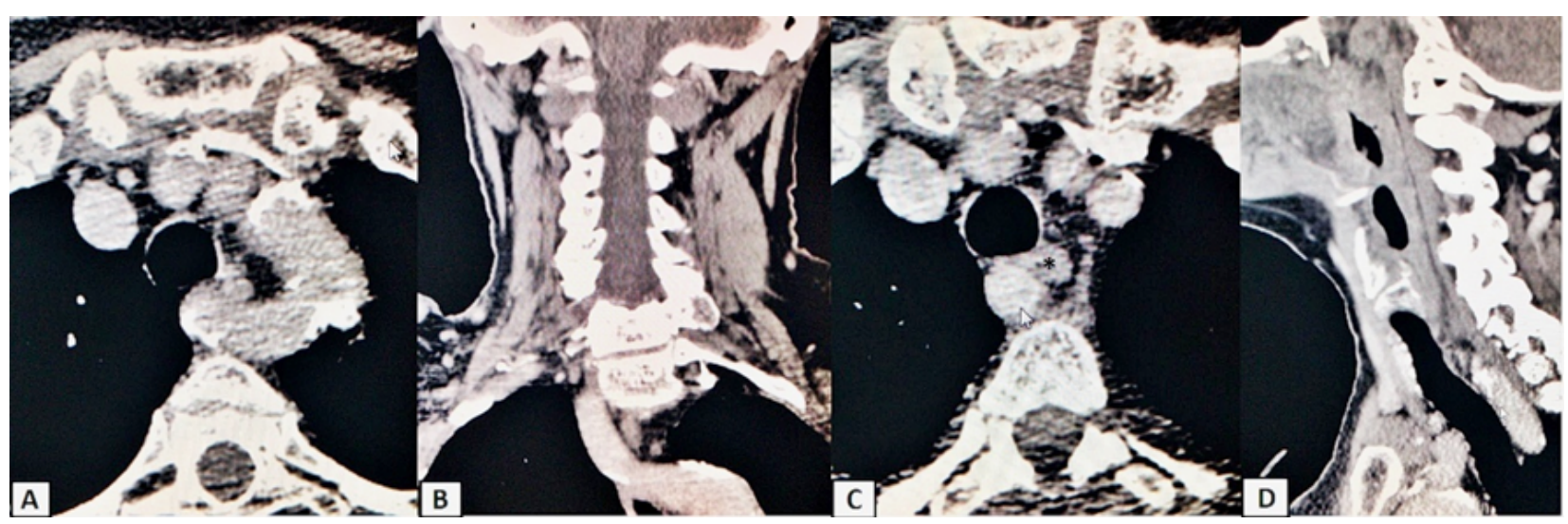

Figure 1: A contrast enhanced Computed tomography showed an aberrant right subclavian artery, originating distally to the origin of the left subclavian artery on axial view (Panel A) and on a coronal view (Panel B), with no signs of aneurysmatic dilatation. Its course to its usual site runs behind the esophagus (black asterisk, Panel C) on axial view (arrow, Panel C) and on sagittal view (arrow, Panel D).

Hoarseness due to inferior laryngeal nerve palsy caused by cardiovascular disease was first described by Ortner in 1897. As far as we know, there is no case report of a right aberrant retroesophageal subclavian artery without aneurysmatic dilatation associated with left vocal cord palsy. The most similar case to ours was published by Harada et al. in 20014: the case of a 55-year-old man with aortic arch aneurysm and an aneurysm of the aberrant right subclavian artery.

The aberrant or anomalous right subclavian artery assuming this retroesophageal course may cause dysphagia by compressing the esophagus, the latter being called dysphagia Iusoria ${ }^{5}$. This condition was first described by David Bayford in 1761, while reporting a fatal case of 'obstructed deglutition' ${ }^{\text {. The }}$ prevalence of arteria lusoria, based on cadaveric studies, is around 1.2 to $1.3 \% .^{5}$ The aberrant artery may compress the left laryngeal nerve, causing a palsy of that nerve, completing criteria for OS. In this particular case, two unusual situations appear together, which contributes to the rarity of the event.

\section{Informed Consent}

Written informed consent was obtained from the patient discussed participated in this study.

\section{Conflicts of Interest}

No conflict of interest was declared by the authors.

\section{Financial Disclosure}

The authors declared that this study received no financial support.

\section{REFERENCES}

1. Simpson CB, Cheung EJ, Jackson CJ. Vocal fold paresis: clinical and electrophysiologic features in a tertiary laryngology practice.

J Voice. 2009:23(3):396-8.

2. Klee K, Eick C, Witlandt R, Gawaz M, Didczuneit-Sandhop B. Unilateral recurrent nerve palsy and cardiovascular disease Ortner's syndrome. J Cardiol Cases. 2016;15:88-90.
3. Yuan S-M. Ortner (cardio-vocal) syndrome: a collective review. Kuwait Med J. 2014;46:3-13.

4. Harada $\mathrm{H}$, Ito $\mathrm{T}$, Yamamoto $\mathrm{N}$, Abe $\mathrm{T}$. Surgical treatment of an aneurysm of the aberrant right subclavian artery involving an aortic arch aneurysm and coronary artery disease. Ann Thorac Cardiovasc Surg. 2001;7:109-12.
5. Asherson N. David Bayford: his syndrome and sign of dysphagia lusoria. Ann R Coll Surg Engl. 1979;61:63-7.

6. Polednak AP. Prevalence of the aberrant right subclavian artery reported in a published systematic review of cadaveric studies: The impact of an outlier. Clin Anat. 2017;30:1024-8. 